\title{
Quaternary Cocrystals Based on Halide-Binding Foldamers through both Hydrogen and Halogen Bonding
}

Lekai Hou, Lingyan Gao, Wenyao Zhang, Xiao-Juan Yang, Biao Wu*

Key Laboratory of Synthetic and Natural Functional Molecule Chemistry of the Ministry of Education, College of Chemistry and Materials Science, Northwest University, Xi'an 710069 (China)

E-mail: wubiao@nwu.edu.cn

\section{Table of Contents:}

S1. Materials

S2. X-ray crystallography

S3. Thermogravimetric analysis of complexes 1-6

S4. References 


\section{S1. Materials}

All reagents and solvents are commercially available and were used as received. The ligand is synthesized according to previously reported methods. ${ }^{1}$ The $\alpha$-methylcholine iodide ${ }^{2}$ and $N, N, N$-trimethyl- $N$-ethylammonium bromide salt ${ }^{3}$ have been reported in our previous work.

\section{S2. X-ray crystallography}

Diffraction data for 1-6 were collected on a Bruker SMART APEX II diffractometer at 100 $\mathrm{K}$ with graphite-monochromated Mo $\mathrm{K} \alpha$ radiation $(\lambda=0.71073 \AA)$. Empirical absorption correction by using SADABS was applied for all data. ${ }^{4}$ The structures were solved by direct methods using the SHELXT program. ${ }^{5}$ All non-hydrogen atoms were refined anisotropically by full-matrix least-squares on $\mathrm{F}^{2}$ by the use of the SHELXL program. ${ }^{6}$ All hydrogen atoms were placed in calculated positions and refined in the «riding model» with $U$ iso $(\mathrm{H})=1.2 U$ eq $(U$ iso $(\mathrm{H})$ $=1.5 \mathrm{Ueq}$ for the hydrogen atoms in $\mathrm{CH}_{3}$ groups) of their parent atoms .

For compound 3, some remaining solvents could not be successfully resolved despite numerous attempts at modeling, and consequently the SQUEEZE function of PLATON was used to account for these highly disordered solvents. The removed void electron density corresponds to about 3 water molecules. In complex $\mathbf{3}$, DFIX was used to restrain the distance between these atoms for H41B and H42B at 1.80 A. DELU were used to the disordered adjacent atoms for C38 and C39, C44 and C45, O9 and C40. ISOR was used to the disordered atoms for C45 and O10. In complex 5, DFIX were used to restrain the distance between these atoms for $\mathrm{C} 51$ and $\mathrm{Cl} 2$ at 1.79 $\AA$, C52 and $\mathrm{C} 11$ at $1.79 \AA, \mathrm{C} 51$ and $\mathrm{C} 52$ at $1.54 \AA$. In complex 6, ISOR was used to the disordered atom for $\mathrm{N} 4$.

Complex 3 contains a chiral cation (but the whole structure consists of racemic foldamers), and we have tried to solve the structure by using a chiral space group. However, due to the weak diffractions at higher angles, a chiral space group could not be determined (and refined) correctly, so the complex 3 was refined in the achiral space group $P \overline{1}$. CheckCIF of complex 4 gives $\mathrm{B}$ level alerts, which may be due to the intermolecular close packing.

The crystal data and refinement details are given in Table S1. Hydrogen bond information for complex 1-6 is given in Tables S2-S7. CCDC numbers are 2053575-2053580. 
Table S1. Crystallographic Data and Refinement Details for Complexes 1-6.

\begin{tabular}{llll}
\hline Complex & $\mathbf{1}$ & $\mathbf{2}$ & $\mathbf{3}$ \\
\hline Empirical formula & $\mathrm{C}_{51} \mathrm{H}_{69} \mathrm{Cl}_{5} \mathrm{~N}_{12} \mathrm{O}_{8}$ & $\mathrm{C}_{51} \mathrm{H}_{69} \mathrm{Br}_{2} \mathrm{Cl}_{3} \mathrm{~N}_{12} \mathrm{O}_{8}$ & $\mathrm{C}_{93} \mathrm{H}_{120} \mathrm{I}_{4} \mathrm{Cl}_{6} \mathrm{~N}_{24} \mathrm{O}_{20}$ \\
Formula weight & 1155.43 & 1244.35 & 2614.42 \\
Crystal system & Triclinic & Triclinic & Triclinic \\
Space group & $P \overline{1}$ & $P \overline{1}$ & $P \overline{1}$ \\
$a(\AA)$ & $13.052(3)$ & $12.9520(5)$ & $11.321(3)$ \\
$b(\AA)$ & $15.681(3)$ & $15.6253(6)$ & $13.975(3)$ \\
$c(\AA)$ & $16.640(3)$ & $16.4996(6)$ & $20.366(5)$ \\
$\alpha($ deg$)$ & $110.961(2)$ & $111.5040(10)$ & $103.516(3)$ \\
$\beta($ deg$)$ & $98.440(3)$ & $98.4770(10)$ & $98.240(3)$ \\
$\gamma($ deg$)$ & $107.496(3)$ & $106.7690(10)$ & $105.910(3)$ \\
$V\left(\AA \AA^{3}\right)$ & $2907.0(9)$ & $2851.97(19)$ & $2937.8(12)$ \\
$Z$ & 2 & 2 & 1 \\
$D_{\text {calc, }}$ g/cm & & 1.449 & 1.478 \\
No. of unique data & 1.320 & 10430 & 10502 \\
$T(\mathrm{~K})$ & 10382 & $100(2)$ & $100(2)$ \\
Total no. of data & $100(2)$ & 78812 & 19455 \\
Crystal size (mm) & 19511 & $0.30 \times 0.30 \times 0.30$ & $0.30 \times 0.3 \times 0.20$ \\
$\theta$ range & $0.40 \times 0.30 \times 0.30$ & 1.813 to 25.368 & 1.918 to 25.454 \\
Completeness to $\theta$ & 1.505 to 25.401 & $99.8 \%$ & $96.7 \%$ \\
Max. and min. transmission & $97.6 \%$ & 0.7452 and 0.4925 & 0.7452 and 0.6049 \\
Goodness-of-fit on $F^{2}$ & 0.7452 and 0.6825 & 1.037 & 1.045 \\
$R 1$ & 0.951 & 0.0242 & 0.0868 \\
$w R 2$ & 0.0587 & 0.0860 & 0.2495 \\
CCDC number & 0.1450 & 2053576 & 2053577 \\
\hline & 2053575 & &
\end{tabular}

\begin{tabular}{llll}
\hline Complex & $\mathbf{4}$ & $\mathbf{5}$ & $\mathbf{6}$ \\
\hline Empirical formula & $\mathrm{C}_{51} \mathrm{H}_{69} \mathrm{Br}_{5} \mathrm{~N}_{12} \mathrm{O}_{8}$ & $\mathrm{C}_{52} \mathrm{H}_{72} \mathrm{Br}_{2} \mathrm{Cl}_{2} \mathrm{~N}_{12} \mathrm{O}_{8}$ & $\mathrm{C}_{51} \mathrm{H}_{70} \mathrm{Br}_{4} \mathrm{~N}_{12} \mathrm{O}_{8}$ \\
Formula weight & 1377.73 & 1223.93 & 1298.83 \\
Crystal system & Triclinic & Monoclinic & Triclinic \\
Space group & $P \overline{1}$ & $P 2(1) / n$ & $P \overline{1}$ \\
$a(\AA)$ & $12.9911(13)$ & $18.628(8)$ & $13.085(3)$ \\
$b(\AA)$ & $15.7215(15)$ & $13.667(6)$ & $15.628(3)$ \\
$c(\AA)$ & $16.4109(16)$ & $24.907(11)$ & $16.507(3)$ \\
$\alpha(\operatorname{deg})$ & $111.0750(10)$ & 90 & $111.696(2)$ \\
$\beta(\operatorname{deg})$ & $97.8390(10)$ & $106.937(6)$ & $102.425(3)$ \\
$\gamma(\mathrm{deg})$ & $106.3250(10)$ & 90 & $105.167(3)$ \\
$V\left(\AA^{3}\right)$ & $2894.0(5)$ & $6066(5)$ & $2838.1(10)$ \\
$Z$ & 2 & 4 & 2
\end{tabular}




\begin{tabular}{llll}
$D_{\text {calc, }} \mathrm{g} / \mathrm{cm}^{3}$ & 1.581 & 1.340 & 1.520 \\
No. of unique data & 10284 & 11714 & 10049 \\
$T(\mathrm{~K})$ & $100(2)$ & $100(2)$ & $100(2)$ \\
Total no. of data & 19232 & 40635 & 18307 \\
Crystal size $(\mathrm{mm})$ & $0.30 \times 0.30 \times 0.20$ & $0.40 \times 0.30 \times 0.30$ & $0.40 \times 0.30 \times 0.20$ \\
$\theta$ range & 1.486 to 25.377 & 1.614 to 26.024 & 1.416 to 25.315 \\
Completeness to $\theta$ & $97.1 \%$ & $99.8 \%$ & $97.3 \%$ \\
Max. and min. transmission & 0.7452 and 0.5910 & 0.7452 and 0.5840 & 0.7452 and 0.3773 \\
Goodness-of-fit on $F^{2}$ & 0.945 & 1.263 & 1.119 \\
$R 1$ & 0.0398 & 0.0690 & 0.0505 \\
$w R 2$ & 0.1043 & 0.1917 & 0.1538 \\
CCDC number & 2053578 & 2053579 & 2053580 \\
\hline
\end{tabular}

Table S2. Hydrogen bond parameters in crystal structure of complex $\mathbf{1}$.

\begin{tabular}{ccccc}
\hline $\mathrm{D}-\mathrm{H} \cdots \mathrm{A}$ & $\mathrm{d}(\mathrm{D}-\mathrm{H})$ & $\mathrm{d}(\mathrm{H} \cdots \mathrm{A})$ & $\mathrm{d}(\mathrm{D} \cdots \mathrm{A})$ & $\angle(\mathrm{DHA})$ \\
\hline $\mathrm{N} 2-\mathrm{H} 2 \cdots \mathrm{Cl} 2$ & 0.88 & 2.40 & $3.263(4)$ & 165 \\
$\mathrm{~N} 3-\mathrm{H} 3 \mathrm{~A} \cdots \mathrm{Cl} 2$ & 0.88 & 2.69 & $3.486(4)$ & 151 \\
$\mathrm{~N} 4-\mathrm{H} 4 \cdots \mathrm{Cl} 1$ & 0.88 & 2.35 & $3.223(4)$ & 172 \\
$\mathrm{~N} 5-\mathrm{H} 5 \cdots \mathrm{Cl} 1$ & 0.88 & 2.73 & $3.520(4)$ & 151 \\
$\mathrm{~N} 6-\mathrm{H} 6 \cdots \mathrm{Cl} 2$ & 0.88 & 2.37 & $3.240(3)$ & 169 \\
$\mathrm{~N} 7-\mathrm{H} 7 \cdots \mathrm{Cl} 2$ & 0.88 & 2.71 & $3.472(4)$ & 145 \\
$\mathrm{~N} 8-\mathrm{H} 8 \cdots \mathrm{Cl} 1$ & 0.88 & 2.57 & $3.405(4)$ & 158 \\
$\mathrm{~N} 9-\mathrm{H} 9 \cdots \mathrm{Cl} 1$ & 0.88 & 2.40 & $3.256(4)$ & 165 \\
$\mathrm{C} 19-\mathrm{H} 19 \cdots \mathrm{Cl} 4$ & 0.95 & 2.89 & $3.792(5)$ & 158 \\
$\mathrm{C} 33-\mathrm{H} 33 \cdots \mathrm{Cl} 4$ & 0.95 & 2.86 & $3.805(5)$ & 172 \\
$\mathrm{C} 41-\mathrm{H} 41 \mathrm{~B} \cdots \mathrm{Cl} 2$ & 0.99 & 2.76 & $3.733(5)$ & 169 \\
$\mathrm{C} 46-\mathrm{H} 46 \mathrm{~B} \cdots \mathrm{Cl} 1$ & 0.98 & 2.79 & $3.691(5)$ & 153 \\
$\mathrm{C} 51-\mathrm{H} 51 \cdots \mathrm{O} 3$ & 1.00 & 1.99 & $2.975(6)$ & 167 \\
\hline
\end{tabular}


Table S3. Hydrogen bond parameters in crystal structure of complex 2 .

\begin{tabular}{ccccc}
\hline $\mathrm{D}-\mathrm{H} \cdots \mathrm{A}$ & $\mathrm{d}(\mathrm{D}-\mathrm{H})$ & $\mathrm{d}(\mathrm{H} \cdots \mathrm{A})$ & $\mathrm{d}(\mathrm{D} \cdots \mathrm{A})$ & $\angle(\mathrm{DHA})$ \\
\hline $\mathrm{N} 2-\mathrm{H} 2 \cdots \mathrm{Br} 2$ & 0.88 & 2.52 & $3.374(2)$ & 165 \\
$\mathrm{~N} 3-\mathrm{H} 3 \cdots \mathrm{Br} 2$ & 0.88 & 2.72 & $3.528(2)$ & 154 \\
$\mathrm{~N} 4-\mathrm{H} 4 \cdots \mathrm{Br} 1$ & 0.88 & 2.45 & $3.323(2)$ & 170 \\
$\mathrm{~N} 5-\mathrm{H} 5 \cdots \mathrm{Br} 1$ & 0.88 & 2.78 & $3.577(2)$ & 152 \\
$\mathrm{~N} 6-\mathrm{H} 6 \cdots \mathrm{Br} 2$ & 0.88 & 2.46 & $3.318(2)$ & 165 \\
$\mathrm{~N} 7-\mathrm{H} 7 \cdots \mathrm{Br} 2$ & 0.88 & 2.87 & $3.568(2)$ & 138 \\
$\mathrm{~N} 8-\mathrm{H} 8 \cdots \mathrm{Br} 1$ & 0.88 & 2.69 & $3.521(2)$ & 159 \\
$\mathrm{~N} 9-\mathrm{H} 9 \cdots \mathrm{Br} 1$ & 0.88 & 2.48 & $3.351(2)$ & 168 \\
$\mathrm{C} 19-\mathrm{H} 19 \cdots \mathrm{Cl} 3$ & 0.95 & 2.87 & $3.758(2)$ & 156 \\
$\mathrm{C} 33-\mathrm{H} 33 \cdots \mathrm{Cl} 3$ & 0.95 & 2.87 & $3.816(2)$ & 174 \\
$\mathrm{C} 35-\mathrm{H} 35 \mathrm{~A} \cdots \mathrm{Br} 2$ & 0.99 & 2.82 & $3.796(2)$ & 170 \\
$\mathrm{C} 50-\mathrm{H} 50 \mathrm{~B} \cdots \mathrm{Br} 1$ & 0.98 & 2.88 & $3.759(2)$ & 149 \\
$\mathrm{C} 51-\mathrm{H} 51 \cdots \mathrm{O} 3$ & 1.00 & 2.01 & $2.990(3)$ & 167 \\
\hline
\end{tabular}

Table S4. Hydrogen bond parameters in crystal structure of complex 3 .

\begin{tabular}{|c|c|c|c|c|}
\hline $\mathrm{D}-\mathrm{H} \cdots \mathrm{A}$ & $\mathrm{d}(\mathrm{D}-\mathrm{H})$ & $\mathrm{d}(\mathrm{H} \cdots \mathrm{A})$ & $\mathrm{d}(\mathrm{D} \cdots \mathrm{A})$ & $\angle$ (DHA) \\
\hline $\mathrm{N} 2-\mathrm{H} 2 \cdots \mathrm{I} 1$ & 0.88 & 2.78 & $3.608(8)$ & 157 \\
\hline N3-H3A $\cdots$ I1 & 0.88 & 3.03 & $3.830(8)$ & 152 \\
\hline $\mathrm{N} 4-\mathrm{H} 4 \cdots \mathrm{I} 2$ & 0.88 & 2.78 & $3.629(8)$ & 162 \\
\hline N5-H5A $\cdots$ I2 & 0.88 & 2.73 & $3.589(6)$ & 167 \\
\hline N6-H6A $\cdots$ I1 & 0.88 & 2.88 & $3.713(7)$ & 158 \\
\hline $\mathrm{N} 7-\mathrm{H} 7 \cdots \mathrm{I} 1$ & 0.88 & 2.89 & $3.687(7)$ & 151 \\
\hline $\mathrm{N} 8-\mathrm{H} 8 \cdots \mathrm{I} 2$ & 0.88 & 2.91 & $3.746(7)$ & 159 \\
\hline N9-H9A $\cdots$ I2 & 0.88 & 2.68 & $3.550(8)$ & 172 \\
\hline C35-H35A $\cdots$ I1 & 0.98 & 3.15 & $4.065(13)$ & 156 \\
\hline C37-H37B $\cdots$ I1 & 0.98 & 3.36 & $4.202(13)$ & 145 \\
\hline $\mathrm{C} 41-\mathrm{H} 41 \mathrm{C} \cdots \mathrm{I} 2$ & 0.98 & 3.28 & $4.203(17)$ & 157 \\
\hline $\mathrm{C} 44-\mathrm{H} 44 \cdots \mathrm{I} 2$ & 1.00 & 3.30 & $4.182(13)$ & 148 \\
\hline O10-HA $\cdots$ O5 & 0.84 & 2.13 & $2.931(17)$ & 161 \\
\hline
\end{tabular}


Table S5. Hydrogen bond parameters in crystal structure of complex 4.

\begin{tabular}{ccccc}
\hline $\mathrm{D}-\mathrm{H} \cdots \mathrm{A}$ & $\mathrm{d}(\mathrm{D}-\mathrm{H})$ & $\mathrm{d}(\mathrm{H} \cdots \mathrm{A})$ & $\mathrm{d}(\mathrm{D} \cdots \mathrm{A})$ & $\angle(\mathrm{DHA})$ \\
\hline $\mathrm{N} 2-\mathrm{H} 2 \mathrm{~A} \cdots \mathrm{Br} 2$ & 0.88 & 2.56 & $3.413(5)$ & 165 \\
$\mathrm{~N} 3-\mathrm{H} 3 \mathrm{~A} \cdots \mathrm{Br} 2$ & 0.88 & 2.72 & $3.535(5)$ & 155 \\
$\mathrm{~N} 4-\mathrm{H} 4 \cdots \mathrm{Br} 1$ & 0.88 & 2.47 & $3.338(4)$ & 168 \\
$\mathrm{~N} 5-\mathrm{H} 5 \mathrm{~A} \cdots \mathrm{Br} 1$ & 0.88 & 2.74 & $3.542(4)$ & 153 \\
$\mathrm{~N} 6-\mathrm{H} 6 \mathrm{~A} \cdots \mathrm{Br} 2$ & 0.88 & 2.51 & $3.367(4)$ & 166 \\
$\mathrm{~N} 7-\mathrm{H} 7 \cdots \mathrm{Br} 2$ & 0.88 & 2.78 & $3.510(4)$ & 141 \\
$\mathrm{~N} 8-\mathrm{H} 8 \cdots \mathrm{Br} 1$ & 0.88 & 2.70 & $3.537(4)$ & 159 \\
$\mathrm{~N} 9-\mathrm{H} 9 \mathrm{~A} \cdots \mathrm{Br} 1$ & 0.88 & 2.51 & $3.371(4)$ & 167 \\
$\mathrm{C} 19-\mathrm{H} 19 \cdots \mathrm{Br} 5$ & 0.95 & 2.95 & $3.885(5)$ & 167 \\
$\mathrm{C} 33-\mathrm{H} 33 \cdots \mathrm{Br} 5$ & 0.95 & 2.96 & $3.911(6)$ & 178 \\
$\mathrm{C} 36-\mathrm{H} 36 \mathrm{~B} \cdots \mathrm{Br} 1$ & 0.98 & 2.88 & $3.762(5)$ & 150 \\
$\mathrm{C} 44-\mathrm{H} 44 \mathrm{C} \cdots \mathrm{Br} 2$ & 0.98 & 3.01 & $3.916(4)$ & 154 \\
$\mathrm{C} 48-\mathrm{H} 48 \mathrm{~A} \cdots \mathrm{Br} 2$ & 0.98 & 3.03 & $3.716(5)$ & 129 \\
$\mathrm{C} 49-\mathrm{H} 49 \mathrm{~B} \cdots \mathrm{Br} 2$ & 0.99 & 2.87 & $3.856(5)$ & 172 \\
$\mathrm{C} 51-\mathrm{H} 51 \cdots \mathrm{O} 3$ & 1.00 & 2.01 & $3.014(8)$ & 177 \\
\hline
\end{tabular}

Table S6. Hydrogen bond parameters in crystal structure of complex 5.

\begin{tabular}{ccccc}
\hline $\mathrm{D}-\mathrm{H} \cdots \mathrm{A}$ & $\mathrm{d}(\mathrm{D}-\mathrm{H})$ & $\mathrm{d}(\mathrm{H} \cdots \mathrm{A})$ & $\mathrm{d}(\mathrm{D} \cdots \mathrm{A})$ & $\angle(\mathrm{DHA})$ \\
\hline $\mathrm{N} 2-\mathrm{H} 2 \cdots \mathrm{Br} 2$ & 0.88 & 2.59 & $3.434(4)$ & 162 \\
$\mathrm{~N} 3-\mathrm{H} 3 \cdots \mathrm{Br} 2$ & 0.88 & 2.59 & $3.440(4)$ & 163 \\
$\mathrm{~N} 4-\mathrm{H} 4 \cdots \mathrm{Br} 1$ & 0.88 & 2.62 & $3.470(3)$ & 163 \\
$\mathrm{~N} 5-\mathrm{H} 5 \cdots \mathrm{Br} 1$ & 0.88 & 2.60 & $3.454(4)$ & 164 \\
$\mathrm{~N} 6-\mathrm{H} 6 \cdots \mathrm{Br} 2$ & 0.88 & 2.69 & $3.529(3)$ & 160 \\
$\mathrm{~N} 7-\mathrm{H} 7 \cdots \mathrm{Br} 2$ & 0.88 & 2.59 & $3.424(3)$ & 158 \\
$\mathrm{~N} 8-\mathrm{H} 8 \cdots \mathrm{Br} 1$ & 0.88 & 2.80 & $3.615(3)$ & 156 \\
$\mathrm{~N} 9-\mathrm{H} 9 \cdots \mathrm{Br} 1$ & 0.88 & 2.47 & $3.330(4)$ & 165 \\
$\mathrm{C} 35-\mathrm{H} 35 \mathrm{~A} \cdots \mathrm{Br} 2$ & 0.99 & 2.86 & $3.833(7)$ & 168 \\
$\mathrm{C} 44-\mathrm{H} 44 \mathrm{~B} \cdots \mathrm{Br} 1$ & 0.99 & 2.99 & $3.949(6)$ & 163 \\
$\mathrm{C} 51-\mathrm{H} 51 \mathrm{~A} \cdots \mathrm{O} 4$ & 0.99 & 2.28 & $3.264(2)$ & 170 \\
$\mathrm{C} 51-\mathrm{H} 51 \mathrm{~B} \cdots \mathrm{O} 6$ & 0.99 & 2.60 & $3.578(2)$ & 168 \\
\hline
\end{tabular}


Table S7. Hydrogen bond parameters in crystal structure of complex 6.

\begin{tabular}{ccccc}
\hline $\mathrm{D}-\mathrm{H} \cdots \mathrm{A}$ & $\mathrm{d}(\mathrm{D}-\mathrm{H})$ & $\mathrm{d}(\mathrm{H} \cdots \mathrm{A})$ & $\mathrm{d}(\mathrm{D} \cdots \mathrm{A})$ & $\angle(\mathrm{DHA})$ \\
\hline $\mathrm{N} 2-\mathrm{H} 2 \cdots \mathrm{Br} 1$ & 0.88 & 2.50 & $3.367(3)$ & 168 \\
$\mathrm{~N} 3-\mathrm{H} 3 \cdots \mathrm{Br} 1$ & 0.88 & 2.86 & $3.656(3)$ & 151 \\
$\mathrm{~N} 4-\mathrm{H} 4 \cdots \mathrm{Br} 2$ & 0.88 & 2.50 & $3.372(4)$ & 172 \\
$\mathrm{~N} 5-\mathrm{H} 5 \cdots \mathrm{Br} 2$ & 0.88 & 2.82 & $3.621(4)$ & 153 \\
$\mathrm{~N} 6-\mathrm{H} 6 \cdots \mathrm{Br} 1$ & 0.88 & 2.59 & $3.434(3)$ & 162 \\
$\mathrm{~N} 7-\mathrm{H} 7 \cdots \mathrm{Br} 1$ & 0.88 & 2.99 & $3.686(4)$ & 138 \\
$\mathrm{~N} 8-\mathrm{H} 8 \cdots \mathrm{Br} 2$ & 0.88 & 2.70 & $3.536(4)$ & 160 \\
$\mathrm{~N} 9-\mathrm{H} 9 \cdots \mathrm{Br} 2$ & 0.88 & 2.55 & $3.409(4)$ & 166 \\
$\mathrm{C} 19-\mathrm{H} 19 \cdots \mathrm{Br} 4$ & 0.95 & 3.11 & $3.901(4)$ & 142 \\
$\mathrm{C} 33-\mathrm{H} 33 \cdots \mathrm{Br} 4$ & 0.95 & 3.00 & $3.907(5)$ & 160 \\
$\mathrm{C} 39-\mathrm{H} 39 \mathrm{~B} \cdots \mathrm{Br} 2$ & 0.99 & 3.03 & $3.813(4)$ & 136 \\
$\mathrm{C} 46-\mathrm{H} 46 \mathrm{~A} \cdots \mathrm{Br} 3$ & 0.98 & 3.03 & $3.918(5)$ & 151 \\
$\mathrm{C} 46-\mathrm{H} 46 \mathrm{C} \cdots \mathrm{Br} 1$ & 0.98 & 2.89 & $3.837(5)$ & 162 \\
$\mathrm{C} 47-\mathrm{H} 47 \mathrm{~B} \cdots \mathrm{Br} 1$ & 0.99 & 2.79 & $3.741(4)$ & 161 \\
$\mathrm{C} 51-\mathrm{H} 51 \mathrm{~B} \cdots \mathrm{Br} 1$ & 0.99 & 2.91 & $3.865(5)$ & 162 \\
$\mathrm{C} 51-\mathrm{H} 51 \mathrm{~A} \cdots \mathrm{O} 3$ & 0.99 & 2.32 & $3.126(6)$ & 138 \\
\hline
\end{tabular}

\section{S3. Thermogravimetric analysis of complexes 1-6}

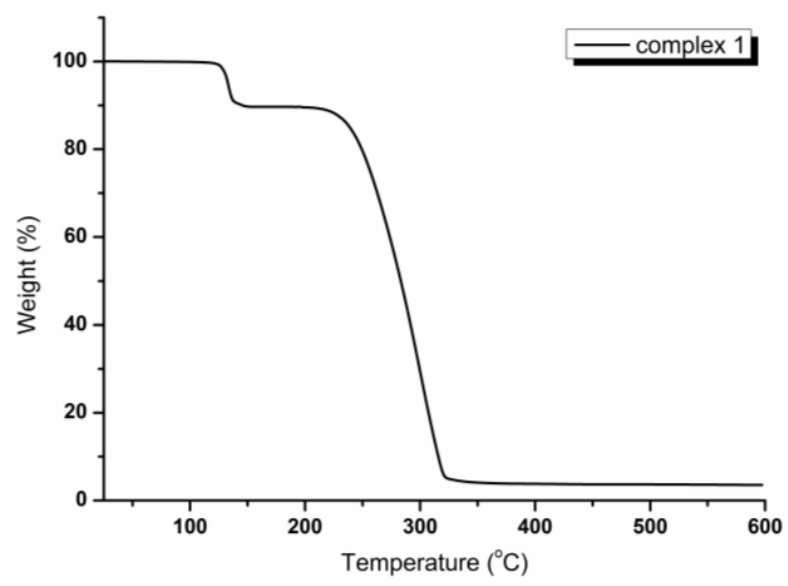

Figure S1. TGA curve of complex 1. 


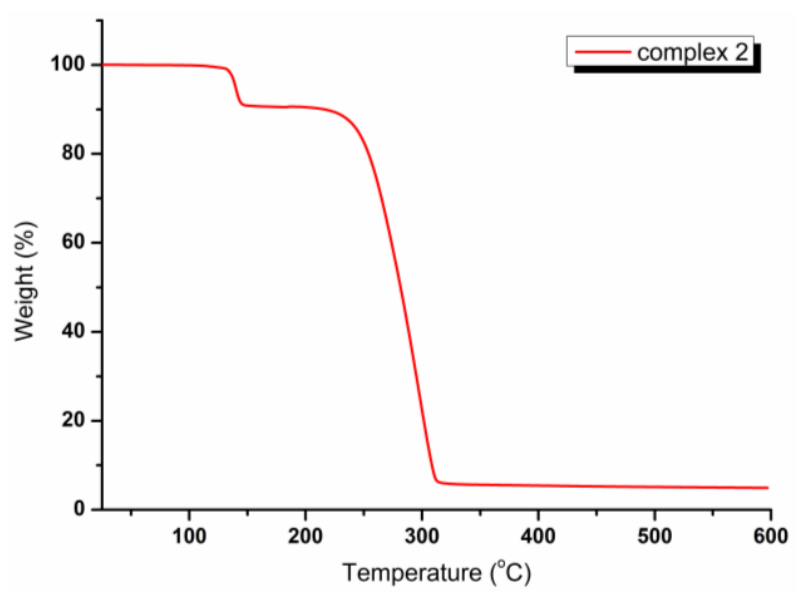

Figure S2 TGA curve of complex 2.

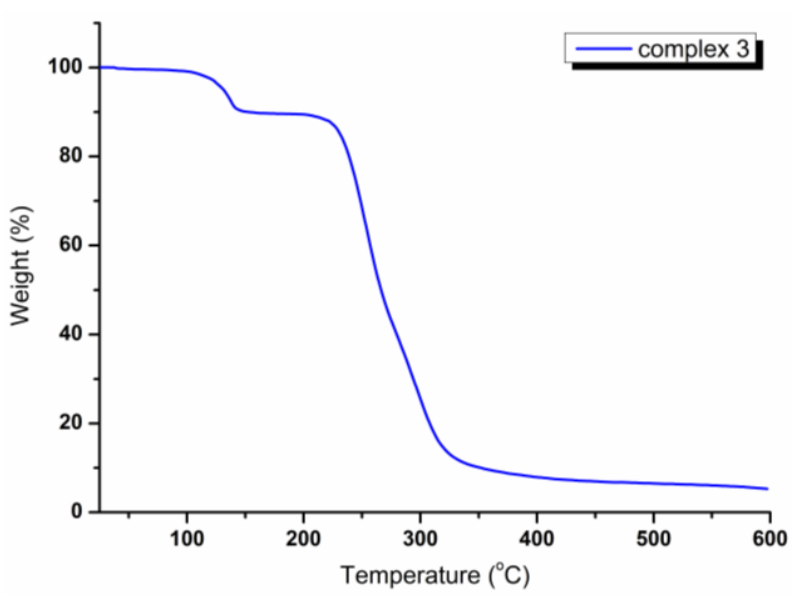

Figure S3. TGA curve of complex 3 .

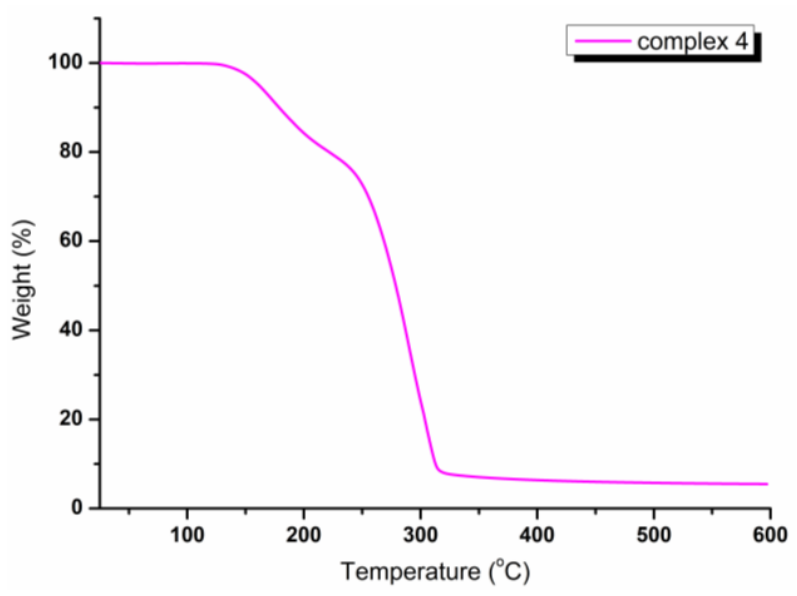

Figure S4. TGA curve of complex 4. 


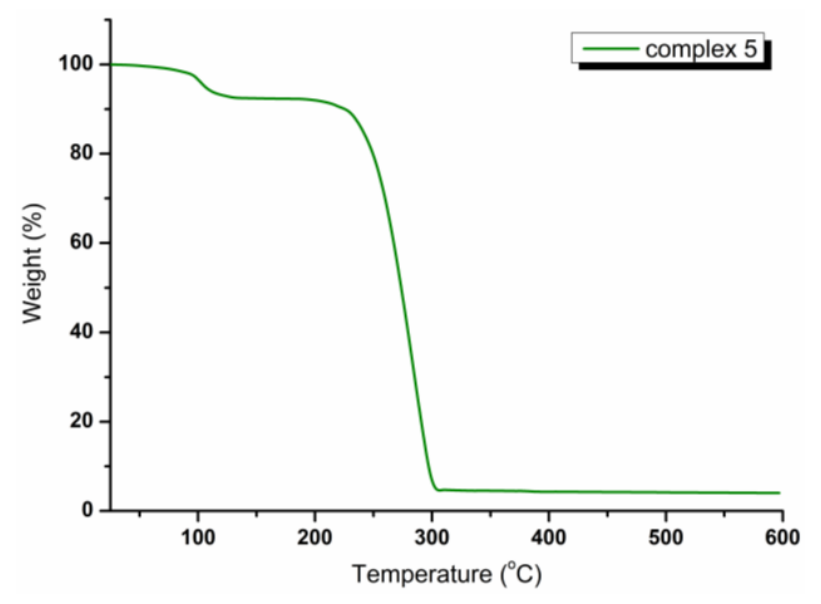

Figure S5. TGA curve of complex 5.

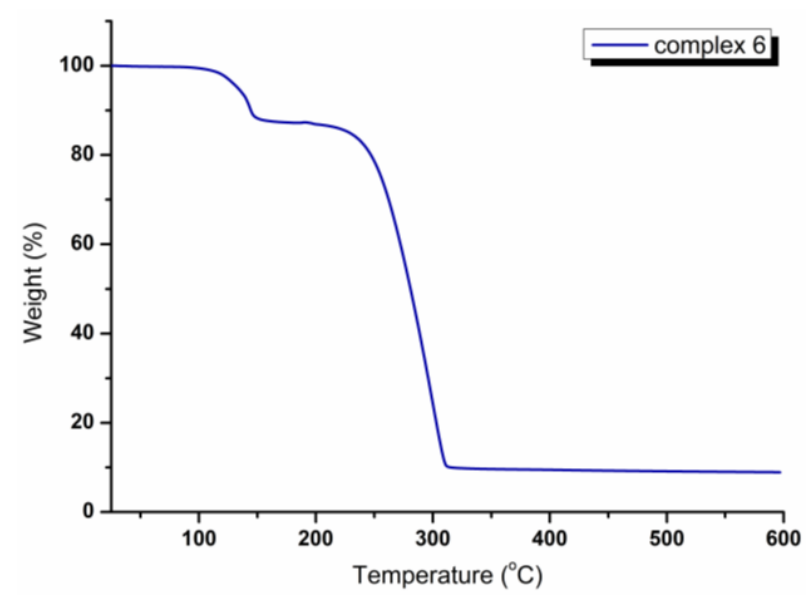

Figure S6. TGA curve of complex 6.

\section{References}

1. Wu, B.; Jia, C.; Wang, X.; Li, S.; Huang, X.; Yang, X.-J. Chloride Coordination by Oligoureas: From Mononuclear Crescents to Dinuclear Foldamers. Org. Lett. 2012, 14, 684-687.

2. Zuo, W.; Huang, Z.; Zhao, Y.; Xu, W.; Liu, Z.; Yang, X.-J.; Jia, C.; Wu, B. Chirality sensing of choline derivatives by a triple anion helicate cage through induced circular dichroism. Chem. Commun. 2018, 54, 7378-7381.

3. Zhang, W.; Yang, D.; Zhao, J.; Hou, L.; Sessler, J. L.; Yang, X.-J.; Wu, B. Controlling the Recognition and Reactivity of Alkyl Ammonium Guests Using an Anion Coordination-Based Tetrahedral Cage. J. Am. Chem. Soc. 2018, 140, 5248-5256.

4. Sheldrick, G. M. Program SADABS: Area-Detector Absorption Correction; University of Göttingen: Göttingen, Germany, 1996.

5. Sheldrick, G. M. SHELXT-Integrated space-group and crystal-structure determination. Acta Crystallogr, Sect. A: Found. Adv. 2015, 71, 3-8.

6. Sheldrick, G. M. Crystal structure refinement with SHELXL. Acta Crystallogr., Sect. C: Struct. Chem. 2015, $71,3-8$. 